Morrison School of Agribusiness and Resource Management

\author{
Faculty Working Paper Series
}

\title{
Pricing Weather Derivatives
}

\section{Timothy J. Richards, Mark R. Manfredo and Dwight R. Sanders}

February 24, 2004

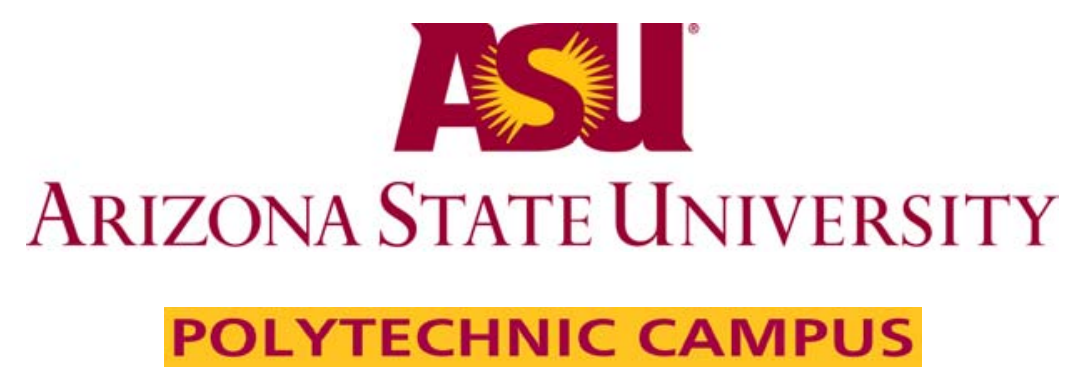




\section{Pricing Weather Derivatives}

by

Timothy J. Richards, Mark R. Manfredo and Dwight R. Sanders

Final Revision: February 24, 2004 


\section{Pricing Weather Derivatives}

Timothy J. Richards, Mark R. Manfredo, and Dwight R. Sanders

Key words: derivative, jump-diffusion process, mean-reversion, volatility, weather

This paper presents a general method for pricing weather derivatives. Specification tests find that a temperature series for Fresno, California follows a mean-reverting Brownian motion process with discrete jumps and ARCH errors. Based on this process, we define an equilibrium pricing model for cooling degree day weather options. Comparing option prices estimated with three methods: a traditional burn-rate approach, a Black-ScholesMerton approximation, and an equilibrium Monte Carlo simulation reveals significant differences. Equilibrium prices are preferred on theoretical grounds, so are used to demonstrate the usefulness of weather derivatives as risk management tools for California specialty crop growers.

Timothy J. Richards is Power Professor of Agribusiness, and Mark R. Manfredo is Assistant Professor, Morrison School of Agribusiness and Resource Management, Arizona State University East. Dwight R. Sanders is Assistant Professor, Department of Agribusiness Economics, Southern Illinois University. The authors wish to acknowledge helpful comments from Cal Turvey, Wade Brorsen and participants in the NCR 134 Applied Commodity Price Analysis, Forecasting, and Market Risk Management Conference, April 2003, St. Louis, Missouri. Funding from the NRI-USDA/CSREES grant number 202-10487 is gratefully acknowledged. 


\section{Pricing Weather Derivatives}

Weather derivatives are contingent securities that promise payment to the holder based on the difference between an underlying weather index - accumulated snowfall, rainfall, or “degree days" over a specified period - and an agreed strike value. ${ }^{1}$ Because weather represents a common source of volume risk for agribusinesses of all types, weather derivatives are a potentially valuable tool for risk management. Compared to insurance contracts, there are many benefits to using weather derivatives to manage risk. First, in order to claim a loss under an insurance contract, a grower must prove that a loss occurred on his or her own farm, or county in the case of area-based insurance products. Adjusting crop losses is expensive to administer and contains an element of subjectivity that growers seldom appreciate. Second, insurance in general is intended to cover the damage caused by infrequent, high-loss events rather than relatively high-probability, limited-loss events. Third, crop insurance products that are based on individual-firm losses are subject to moral hazard problems, so an alternative tool that pays out based on some objective measure of the weather itself may be a preferable alternative (Yoo; Turvey; Cao and Wei). ${ }^{2}$

In spite of these advantages, and the increasing interest in weather risk management more generally (Weather Risk Management Association), the volume of trade in weather derivatives has been growing relatively slowly (Dischel). Several factors contribute to this lack of liquidity, including (1) the absence of a forward market in a relevant weather index, (2) potential basis risk, (3) problems defining meaningful weather data, and (4) the lack of agreement over a common pricing model (Dischel; Nelken; 
Turvey). Although the Chicago Mercantile Exchange (CME) began trading degree-day futures and options for a number of major U.S. cities in the fall of 1999, the fact that weather is a local phenomenon and micro-climates often differ radically within small geographic areas means that the $\mathrm{CME}$ products are of little use to most agricultural producers, or of limited use to many. Second, basis risk is likely to be a significant problem for firms that wish to hedge using derivatives based on weather indices. Basis risk, in this case, refers to the difference between a weather index value defined for a particular location, a large city for example, and the actual value of the same weather index that applies to a specific firm. ${ }^{3}$ Third, without a traded instrument to form part of a riskless hedge, conventional preference-free Black-Scholes (BS) pricing models cannot be used and derivatives must be priced so as to reflect the market price of risk. Although economic research can do little to remedy the first two problems, the third can be addressed by designing an appropriate equilibrium pricing model and demonstrating how it can be applied to a specific type of weather derivative.

The objective of this article, therefore, is to develop an appropriate pricing model for valuing weather derivatives that is sufficiently practical to be useful to agricultural risk managers, yet sufficiently general to be of use to a broad scope of non-agribusiness firms with weather-related risks. By calculating equilibrium prices for cooling-degreeday (CDD) put and call options in a representative production region, the study demonstrates how weather derivatives can be priced. Further, estimates of the temperature - yield relationship for a typical crop show how derivatives can be used by a firm involved in producing and trading an example commodity to effectively manage weather risk. The article begins by briefly explaining how weather derivatives work. 
The second section describes the data used, while the third section presents an equilibrium pricing model based on the general equilibrium approach of Lucas. The fourth section defines the stochastic processes governing weather and aggregate economic output and how the pricing model applies to a particular agricultural region. The prices implied by this model, as well as estimates of the market price of risk and an assessment of the potential hedging effectiveness of weather derivatives are presented and discussed in a fifth section. A final section concludes and offers some suggestions for both future research and product development.

\section{Background on Weather Derivatives}

Weather derivatives exist as either futures, options or swaps and are traded either on formal exchanges (futures and options on the CME) or over-the-counter (options and swaps). Derivatives can be written for any one of a number of weather phenomena, including accumulations of CDD or HDD, a particular sequence of events, such as three consecutive days below 32 degrees Fahrenheit, or cumulative precipitation over a specified period of time. Because temperature derivatives are the most common, this study focuses on options written on either a CDD or HDD index. There are five essential elements to every weather contract: (1) the underlying weather index, (2) the period over which the index accumulates, typically a season or month, (3) the weather station that reports daily maximum and minimum temperatures, (4) the dollar value attached to each move of the index value, and (5) the reference or "strike" value of the underlying index (Cao and Wei). Using a CDD call as an example, the option will acquire value when the cumulative CDD index rises above an agreed strike level. At the agreed expiry date, the 
holder receives a payment if the CDD index rises above the strike level. The amount of the payment is equal to the CDD index less the strike level multiplied by some notional dollar value per unit of the index.

Ideally, the buyer of the derivative is thus compensated by the writer for an amount that offsets the real business losses from adverse weather. For example, an amusement park owner would buy a CDD put that pays out if there is a string of unusually cold days. The value accumulated with the long put position will help offset the lost revenue from customers who have stayed away during the cool weather period. If, on the other hand, the intervening period was unusually hot so that the CDD index rises well above the strike level, then the put expires worthless. The amusement park owner is out the premium paid at the initiation of the contract to the writer of the put, but has likely met desired risk management goals because increased business revenue likely more than compensates for the price of this "insurance policy." A farmer's interest in weather derivatives is analogous to the amusement park owner example. A fruit grower, for example, would likely buy a CDD call so that he or she is compensated if sustained, unusually hot weather during the critical growing period reduces yield.

\section{Weather Data}

\section{Data Description and Sources}

The weather data are from the U.S. National Climatic Data Center (NCDC) for a weather station located in Fresno, CA. Estimates of the temperature data process are obtained using 30 years of daily average temperatures. Although there are many more years of data from the NCDC, the sample used in this study consists of 30 years in order to gain as 
much estimation efficiency as possible while minimizing the "heat island" effect that arises in arid and semi-arid areas with the creation of heat-retaining buildings, roads and artificial parks (Dischel). The weather data consist of daily maximum, minimum, and average temperature as well as daily precipitation for the entire year. Therefore, the weather process itself is estimated using all daily observations in the data set, but the particular CDD index used in the pricing model below describes only the May through July window. Specifically, the CDD index is defined as the cumulative sum of the extent to which daily average temperatures exceed a 65 degree Fahrenheit benchmark:

$$
C D D=\sum_{t=1}^{T} \max \left(0, w_{t}-65\right)
$$

where $w_{\mathrm{t}}$ is the average daily temperature on day $t$ measured in degrees Fahrenheit. Although the temperature series is not directly applicable to any particular grower, primarily because it is gathered at the Fresno Air Terminal, the proximity of many growers to Fresno and the relative topographical homogeneity of the surrounding area should lessen the basis risk for growers farther from the weather station.

Pricing weather derivatives using the general equilibrium framework described below requires an "aggregate dividend," or a measure of aggregate economic activity. In order to minimize basis risk while ensuring that the derivative is relevant to all potential traders in Central California, personal consumption expenditures for Fresno County are chosen for this purpose. These data are from the Bureau of Economic Analysis and are measured in nominal dollars, on an annual basis over the 1970 - 2000 sample period.

Further, to determine the effectiveness of weather derivatives in hedging growers' 
yield risk, it is necessary to estimate the relationship between the CDD index defined above and crop yields. For this purpose, annual county-average yields for an important and representative crop in Fresno County, nectarines, were obtained from the California Department of Food and Agriculture (CDFA) as reported by the Fresno County Agricultural Commissioner's office for the period 1980 - 2001. County-level yield data necessarily induces some aggregation bias, but avoids idiosyncratic yield variations that would arise with farm-level, panel data on crop yields. Table 1 provides summary statistics and basic tests of normality for the average daily temperature data from 1970 2000.

\section{Empirical Model of Weather and Weather Derivatives}

\section{Alternative Stochastic Processes}

When pricing any derivative security, the accuracy of the pricing model depends critically upon the nature of the process for the underlying security or, in this case, the underlying state variable - temperature. In daily data, temperature varies in a way that is relatively well understood (Cao and Wei; Nelken; Turvey; Alaton, Djehiche and Stillberger; West; Yoo). The average daily temperature varies by season, but tends to revert to a long-run average that is likely moving slowly upward with the accumulation of carbon dioxide in the atmosphere. Further, changes in temperature from day to day are not entirely random as weather systems tend to lead to "warm spells" or "cold snaps." Temperature also tends to be more volatile in winter than in summer (Cao and Wei 2002), while rapid changes in temperature are more the rule than the exception. The most general statistical model of the stochastic process for temperature leads to a mean-reverting Brownian motion with 
log-normal jumps and time-varying volatility:

$$
d w_{t}=\kappa\left(\alpha_{w}\left(w_{t}, t\right)-\lambda \phi-w_{t}\right) d t+h_{t}\left(w_{t}, t\right) d z+\phi d q
$$

where $w$ is the average daily temperature in degrees Fahrenheit, $\alpha_{\mathrm{w}}$ is the instantaneous mean of the process, $\kappa$ is the rate of mean-reversion, $h_{\mathrm{w}}$ is the standard deviation, and $d z$ defines the Wiener process with properties: $\mathrm{E}(d z)=0$ and $\mathrm{E}\left(d z^{2}\right)=d t{ }^{4}$ Discrete jumps in temperature occur according to a Poisson process $q$ with average arrival rate $\lambda$ and a random percentage shock, $\phi$, which is distributed log-normal with: $\ln (1+\phi) \sim \mathrm{N}(\gamma-$ $0.5 \delta^{2}, \delta^{2}$ ) where $\delta^{2}$ is the variance of the jump component. The Poisson counter $q$ is distributed as:

$$
d q=\left[\begin{array}{lll}
0 & \text { with probability } 1-\lambda d t \\
1 & \text { with probability } \lambda d t
\end{array}\right]
$$

Accounting for discrete jumps in the weather series this way is relatively common in the literature on stock price fluctuation (Merton; Ball and Torous 1983, 1985; Jarrow and Rosenfeld; Jorion; Bates 1991), exchange rates (Jorion; Naik and Lee; Bates 1996) and commodity prices (Hilliard and Reiss), but is also appropriate for temperature as the killer frost in California in December of 1998 demonstrated.

To accommodate seasonal fluctuations in both the level and volatility of temperature, the mean and variance of (2) are specified as functions of temperature and time. Seasonality, autoregression and trend are removed from the raw temperature series by defining its unconditional mean in a manner similar to Alaton, Djehiche, and Stillberger; Yoo, and West: 


$$
\alpha_{w}\left(w_{t}, t\right)=\gamma_{0}+\gamma_{1} \sin (2 \pi t / 365)+\gamma_{2} \cos (2 \pi t / 365)+\gamma_{3} t+\sum_{j=1}^{p} \rho_{j} w_{t-j}
$$

where the optimal lag is found to be $p=3$ by the Schwarz criterion. Time-varying volatility, on the other hand, is incorporated by specifying the conditional volatility as a first-order autoregressive conditional heteroscedastic $(\mathrm{ARCH})$ process:

$$
h_{t}\left(w_{t}, t\right)=E_{t-1}\left(\sigma_{w t}^{2}\right)=\gamma_{0}+\gamma_{1}\left(w_{t-1}-\alpha_{w}\right)^{2}
$$

the parameters of which are estimated by substituting (5) into the general process (2) and estimating the entire process by maximum likelihood. With an $\mathrm{ARCH}$ specification, shocks can exhibit some memory through volatility, but they are also likely to persist through the mean of the series. Combining each of these elements, the set of alternative

processes to be estimated includes: (1) simple Brownian motion (BM), (2) mean reverting Brownian motion (MRBM), (3) mean reverting Brownian motion with log-normal jumps (MRBM-J) and (4) mean reverting Brownian motion with log-normal jumps and ARCH. The preferred model is selected from among these nested alternatives using a set of paired likelihood ratio tests.

\section{Pricing Model for Weather Derivatives}

Originally, practitioners used simple "burn rate" (BR) models or modified existing BS based pricing models to the weather problem by defining the underlying security in terms of the CME CDD or HDD contracts defined for a specific urban location. ${ }^{5}$ BR models have one key advantage that explains their popularity among practitioners - ease of use. 
However, because there is no way to update the probabilities of adverse weather events, derivatives priced using BR models will trade infrequently, if at all (Dischel; Turvey; Pirrong and Jermakayan; Zeng). No-arbitrage models are also inappropriate because weather is not a traded asset, but rather a state variable, so traders cannot form the riskless hedge on which such models are based. ${ }^{6}$ Further, because the risks associated with weather are non-diversifiable, weather derivative prices must reflect a market price of risk. This rules out the use of a BS type of model. Turvey addresses the absence of a hedgeable asset by applying the risk-neutral pricing model of Cox, Ingersoll and Ross under the simplifying assumptions that the mean drift rate of the weather index and the "weather beta" are both zero. ${ }^{7}$ However, such an approach assumes that the stochastic process governing the HDD index exhibits independent increments. This rules out either mean-reversion or time-varying volatility. On the other hand, Cao and Wei; Davis; Alaton, Djehiche, and Stillberger; and Yoo all provide empirical evidence to the contrary, namely that weather indices are highly non-linear, seasonal and mean-reverting. If this is the case, then there is no stochastic representation that can provide an analytical solution to the valuation problem. Therefore, an alternative approach must be used.

There are three general ways to proceed in the case of incomplete markets: (1) define a risk-neutral equivalent martingale probability measure (EMM) and discount the expected payoff at the risk-free rate (Alaton, Djehiche and Stillberger; Yoo), (2) derive an equilibrium pricing model that explicitly incorporates the market price of risk (Pirrong and Yermakayan), or (3) specify an equilibrium pricing model that implicitly includes a risk premium for the non-traded asset (Cao and Wei). Although the first approach is theoretically valid, we have no way of directly estimating the market price of risk so this 
approach cannot provide a solution to the problem at hand. Neither does the second method include a way of recovering the market price of risk without a forward or futures contract in the underlying weather index. Therefore, this study applies the Lucas general equilibrium valuation model to determine the value of weather derivatives, both put options and call options, using the algorithm developed by Cao and Wei.

Only the components of the Lucas model that are essential to our weather derivative application are described here. The model assumes a pure exchange economy. In other words, in contrast to the endogenous production environment of Cox, Ingersoll and Ross, production is assumed exogenous and agents expected utility maximizers. There are two state variables: (1) production $\left(y_{t}\right)$ and (2) the weather, or more specifically, temperature, $w_{\mathrm{t}}$. Asset prices serve to equilibrate the market for all claims on output given any state of the world and, as such, represent state-dependent contingent claim values. All production in the economy is owned by agents with equity claims that pay an aggregate dividend, $d_{\mathrm{t}}$. The aggregate dividend, in turn, depends on the weather according to an autoregressive process. In equilibrium, consumption is equal to the aggregate dividend. A representative, risk-averse consumer chooses consumption $\left(c_{\mathrm{t}}\right)$ to maximize the present value of expected utility:

$$
e^{-\rho t} E\left[U\left(c_{t}, t\right)\right]=e^{-\rho t} E\left[c^{1-\gamma /(1-\gamma)]}\right.
$$

where $\gamma$ is the constant relative risk aversion (CRRA) parameter, $U_{\mathrm{c}}>0, U_{\mathrm{cc}}<0$ and $0<$ $\gamma<\infty$. Note that $\gamma=0$ corresponds to risk-neutrality and $\gamma=1$ to log-utility, which is a common assumption in models of this type. The value of any contingent claim is found by maximizing the expectation of (6) with respect to consumption subject to the joint 
evolution of the state variables in the model.

While the generating process for weather was defined in equation (2), the aggregate dividend is assumed to follow an autoregressive process where the current period dividend is a function of its value in the previous year as well as current and previous weather innovations:

$$
d_{t}=\alpha_{1} d_{t-1}+\phi_{0} \epsilon_{t}+\phi_{1} \epsilon_{t-1}+\phi_{3} \epsilon_{t-2}+\ldots+\phi_{k} \epsilon_{t-k}+\xi_{t}
$$

where: $\epsilon_{t}=w_{t}-\hat{w}_{t}$ and $\xi_{\mathrm{t}}$ is an iid normal error term. Because the dividend process is a function of both current and previous weather innovations, it provides information on both the contemporaneous and lagged correlation between aggregate output and the weather. This feature is important as it allows us to forecast future values of the dividend based on current realizations of the weather and the weather process estimated above.

Given this process for the dynamic wealth constraint, solving the expected utilitymaximization problem (6) requires the price of any contingent claim to be equal to the dividend ratio at expiry multiplied by its expected payoff. In terms of the objective defined in (6), and substituting in the equilibrium condition $d_{\mathrm{t}}=c_{\mathrm{t}}$, the price of any weather derivative at any date $t$ prior to expiry, $T$, is:

$$
V_{t}=E_{t}\left[e^{-\rho t}\left(\frac{U_{d}\left(d_{T}\right) X_{T}}{U_{d}\left(d_{t}\right)}\right)\right]=e^{-\rho t} d_{t}^{-\gamma} E_{t}\left[d_{T}^{\gamma} X_{T}\right]
$$

where the payoff, $X_{\mathrm{T}}$, to a call option on an underlying CDD index is $\max$ [CDD - $K, 0$ ] for a strike value of $K$. Analogous reasoning holds for a put option. Importantly, note that if the aggregate dividend is not autoregressive and is not correlated with the weather, 
then the derivative price is no longer a function of $d_{\mathrm{t}}$ and $\gamma$, so the market price of risk will equal zero. In this case, the weather derivative price is simply the present value of its expected payoff at expiry. Combining the weather process in (2) and the dividend process in (7) with the valuation model (8) provides the method of valuing weather derivatives.

\section{Estimation and Calibration Procedures}

The first step in implementing the pricing model is to estimate the weather process. There are at least four alternative methods for estimating the parameters of the jump-diffusion weather process given by (2): (1) the method of cumulants (Ball and Torous 1983), (2) direct maximum likelihood estimation (Ball and Torous 1983, 1985; Jorion or Jarrow and Rosenfeld), (3) implied estimation of derivative moments using an existing price series (Hilliard and Reiss) and (4) a least-squares estimator (Bates 1991, 1996). Because there is no weather derivative price series for a weather station in Central California, implicit estimation is not possible, therefore, all models are estimated using maximum likelihood. With this approach, the most general likelihood function is written as: ${ }^{8}$

$$
\begin{aligned}
& L(w \mid \beta)=-T \lambda-\frac{T}{2} \ln (2 \pi)+ \\
& \sum_{t=1}^{T} \ln \left[\sum_{n=0}^{N} \frac{\lambda^{n}}{n !} \frac{1}{\sqrt{h_{t}+\delta^{2} n}} \exp \left(\frac{-\left(v_{t}-\left(\alpha_{0}+h_{t} / 2+n \delta^{2} / 2-n \phi-w_{t}^{a}\right)\right)^{2}}{2\left(h_{t}+\delta^{2} n\right)}\right),\right.
\end{aligned}
$$

for $T$ - 1 observations of discrete changes in the de-trended temperature series: $v_{t}=w_{t}^{a}-w_{t-1}^{a}$, where $w_{\mathrm{t}}^{\mathrm{a}}$ is the temperature residual: $w_{\mathrm{t}}-\alpha_{\mathrm{w}}, \lambda$ is the Poisson intensity parameter, $\delta^{2}$ is the volatility of the discrete part, $h_{\mathrm{t}}$ the volatility of the continuous part, 
and $\phi$ is the mean jump size. ${ }^{9}$ Following Ball and Torous (1985), $n$ is defined as the random realization of a shock to temperature, and $N$ is fixed at a value likely to include all possible occurrences of a shock, and (9) is maximized with respect to the remaining parameters. Likelihood functions for each of the other processes are defined in a similar way, but with appropriate restrictions. As Ball and Torous (1985) suggest, a Bernoulli jump-diffusion model provides useful starting values for the maximum likelihood estimation procedure. With these starting values, convergence of each model occurs within 85 iterations using a Newton-Raphson non-linear solution algorithm with 11,050 daily temperature observations. Because each of the first three models are nested within the fourth, the preferred process is selected on the basis of likelihood ratio tests. Residuals from this process are then calculated for use in the second step - estimating the correlation between weather innovations and the aggregate dividend.

Although it would be preferable to estimate the weather and dividend processes together, doing so is not possible because the weather data are daily, whereas the aggregate dividend is measured on an annual basis. Therefore, the second step involves estimating the dividend process (7) using annualized temperature residuals. A measure of the aggregate dividend must reflect the value of economic output for a particular economy. Therefore, the dividend is defined in terms of aggregate personal consumption expenditure for Fresno county over the 1970 - 2000 time period. Preliminary specification tests of equation (7) found strong evidence of a unit root, so the equation is estimated in first differences. Estimates appear in table 3.

Step three involves calibrating the pricing model and finding prices for both put options and call options on a CDD index for Fresno County. Given the complexity of the 
processes for both weather and the aggregate dividend, finding a closed-form solution to (8) is not possible, so the third step consists of conducting a Monte Carlo simulation of the weather and derivative processes. Finding complex derivative values via Monte Carlo simulation is a generally accepted method (Boyle; Hull; Pirrong and Jermakayan). With this approach, the process for the underlying state variables are simulated a large number of times and the value of the derivative at expiry is calculated using (8). By averaging the implied derivative prices over all random draws, this method provides an estimate of the true derivative price. Specifically, the Monte Carlo simulation constructs 10,000 forward values of the CDD index and, simultaneously, calculates associated values of the aggregate dividend at the expiry date, $T$, using the parameters from the relationship (7). By forecasting the temperature for each day of the sample process, the Monte Carlo simulation provides a distribution of payout values for the derivative at time T. Next, we find the expected value of the product of the payout value and dividend ratio by averaging over all 10,000 draws. Calculating the present value of this average provides an estimate of the derivative price at time $t$.

This framework also provides a convenient method of implicitly estimating the market price of risk. Recall that in the absence of a tradable underlying asset, the market price of risk may have a significant impact on a contingent claim traded in an incomplete market. Indeed, if the market price of risk were known, or could be assumed to be zero, then weather derivatives could be priced using a risk-neutral valuation method (Hull). Without access to a relevant price series for existing weather derivative contracts, estimating the market price against a traded benchmark is impossible. Neither can we assume the market price of risk is zero because, as we demonstrate, aggregate economic 
output and the weather are not independent. Nonetheless, it is possible to estimate the market price of risk as an implicit parameter in a general equilibrium framework. This approach relies on the fact that if there is no correlation (contemporaneous or lagged) between weather and an aggregate market index, then the market price of risk must be zero. ${ }^{10}$ Imposing a zero-correlation assumption on the dividend forecasts calculated using equation (7) produces a lower value for the aggregate dividend at expiry and, hence, a lower derivative value in equilibrium. By comparing derivative values calculated with zero and the positive, or empirical correlation, the market price of risk is simply the annualized percentage difference in the "risk neutral" and the "true" derivative value. Because each draw of the Monte Carlo simulation produces a different estimate of $\lambda$, averaging over all 10,000 draws provides an expected market price of risk that is assumed to be an unbiased estimate of the true value. This procedure is repeated for a range of relative risk aversion parameters, from 0.0 (risk neutrality) to 20.0 (extreme risk aversion) and the market price of risk calculated for each case. Determining an equilibrium price, however, does not guarantee that weather derivatives are valuable risk management tools for agribusiness.

\section{Determining Hedging Effectiveness}

Ultimately, the ability to arrive at an equilibrium price for a derivative is a necessary condition for the development of a liquid market, but is not a sufficient one. Rather, market participants must believe that the derivative represents an effective hedging tool for significant trading volume to arise. Hedging effectiveness, in turn, depends upon the correlation of the underlying state variable, the CDD index in the current example, with 
key measures of economic interest - output, revenue, cost or profit. In the case of an energy producer, Hull argues that the ability to effectively hedge both price and volume risks can be determined by estimating a regression model of his or her profit on power prices and a CDD or HDD index. Finding significant temperature and power-price regression parameters amounts to an empirical "proof" of the likely effectiveness of either a power or weather hedge. For farm commodity producers, analogous reasoning suggests that a regression model of yields on a CDD index calculated over a critical growing period can serve the same purpose. ${ }^{11}$ Using nectarines grown in Fresno County, California as an example, a regression model is estimated that includes a CDD index calculated over the 92-day May to July period when yield potential is determined (c), the square of $c$ and a linear time trend:

$$
y_{i}=\beta_{o}+\beta_{1} c+\beta_{2} c^{2}+\beta_{3} t+\epsilon_{i}
$$

to explain nectarine yields, $y$. Although there are admittedly many other factors that potentially influence yields, if they are uncorrelated with temperature then (10) will provide reliable estimates of the temperature - yield relationship. The results obtained from estimating this model with the Fresno nectarine data are presented and discussed after each of the prior three steps in the algorithm explained here.

\section{Results and Discussion}

This section reports the estimates of all four components of the weather derivative pricing model: (1) maximum likelihood estimates of each alternative CDD process, (2) estimates 
of the aggregate dividend process, (3) Monte Carlo estimates of weather derivative prices under varying degrees of risk aversion, and (4) estimates of hedging effectiveness for a representative agricultural product. Following the presentation of these results, we offer some implications for real-world pricing of weather claims and others for which traditional pricing methods are not available.

Although each set of results contributes to the weather derivative literature, the weather process estimates are likely to be of greatest interest to others searching for a pricing model. Table 2 provides all parameter estimates and likelihood ratio tests used to compare alternative weather process models to the most general one. The results in table 2 indicate rejection of the restricted specifications in favor of the more general model. Therefore, the preferred model is a mean-reverting Brownian motion with log-normal jumps and first-order autoregressive conditional heteroscedastic errors. While timevarying volatility does not allow for random variation in volatility, a battery of goodnessof-fit tests suggest further refinements of this model may not be necessary. For example, under the null hypothesis that the residuals are normally distributed, the Jarque-Bera test statistic is 5.99, resulting in a failure to reject the null at the $5 \%$ level of significance. In addition, a Durbin-Watson test statistic for first-degree autocorrelation lies safely in the region of non-rejection, suggesting that the residuals are approximately white-noise. Consequently, based on the likelihood-ratio test results, the MRBM-J-ARCH model is preferred.

[table 2 in here]

The results in table 2 illustrate sharp qualitative differences in the implications of each process. For example, the simpler BM process dramatically understates the mean 
drift rate. By ignoring mean-reversion, the BM specification implies that the CDD index can wander away from its long-term average indefinitely. This is not likely to happen in reality, so should not be reflected in weather derivative prices. The results in table 2 also show the importance of jumps in the temperature process through the large incremental improvements in fit by each jump-diffusion model relative to its continuous analog. Although the CDD process is physical, rather than financial, these results are consistent with the implications of ignoring "fat tails" in financial asset returns processes found by Bates (1991, 1996); Naik and Lee; and Jorion. Third, although allowing for time-varying volatility improves the fit significantly, including an $\mathrm{ARCH}$ error component is apparently not as important as in other contexts. Specifically, in their comparison of alternative option pricing models, Bakshi, Cao and Chen provide evidence from S\&P 500 index options that allowing for time-varying volatility provides the largest incremental gain in option pricing "fit" from among each of the BS extensions that they consider. By using stocks traded on organized exchanges, however, they are able to use indirect market evidence to demonstrate the superiority of their time-varying volatility models, whereas this study necessarily relies on direct estimation. It remains, however, to determine whether a contingent claim on temperature can be priced in the market.

Indeed, a weather derivative will only have value in a general equilibrium framework if the underlying index is correlated with a measure of aggregate economic output. Table 3 provides evidence of a statistically significant correlation between the weather series residuals and aggregate output both contemporaneously and at lags of one and two periods. Consequently, weather and the aggregate dividend are not independent, so the market price of risk must be taken into account. Although the t-ratios in this 
regression are relatively low, this is not surprising given the short time series available and the number of factors that influence output. Moreover, based on the Durbin-Watson statistic and results from the RESET specification test shown in table 3, this model is well specified.

[table 3 in here]

Table 4 shows equilibrium prices calculated for both at-the-money puts and calls as well as similar derivative prices calculated using traditional BR and BS methods. For the equilibrium prices, this table also provides estimates of the implied market price of risk at each degree of risk aversion and a t-test of the null hypothesis that the price of risk is equal to zero in each case. Several interesting results are apparent from this table.

First, for both puts and calls, and at each level of risk aversion, the market price of risk is significantly different from zero. While it is small in an economic sense at low levels of risk aversion, the market price of risk rises to nearly $30 \%$ of the derivative value for calls, and approximately $11 \%$ for puts. Admittedly, this level of risk aversion is extreme, but it serves to illustrate the potential importance of ignoring risk preferences in estimating derivative prices when a risk neutral valuation method does not apply. While Cao and Wei find a small percentage of their example cities in which the market price of risk is significant, and Alaton similarly concludes that it is likely to be small, the results in table 4 show that the market price of risk is not only statistically significant (relative to the benchmark derivative), but economically large as well. This result is more akin to Pirrong and Jermakayan, who find the market price of risk to be a significant factor in pricing power derivatives.

Note also that the "risk discount" associated with call options is greater in 
absolute value than the premium associated with puts. This result, which is similar to that found by Cao and Wei, reflects the fact that the covariance between the payoff at expiry and the aggregate dividend for a put is negative for a put option and positive for a call. As the dividend ratio $\left(d_{\mathrm{T}} / d_{0}\right)$ is always greater than 1.0 due to the positive correlation between temperature and aggregate output, raising the dividend ratio to a negative exponent in the marginal utility function causes the observed pattern of premiums and discounts. Intuitively, because the expected utility function used in the pricing model is increasing and concave, the marginal utility for a downside temperature movement is greater in absolute value than an equivalent upward movement. As the degree of risk aversion rises, this concavity is accentuated. Consequently, a more riskaverse investor will pay more for the downside protection inherent in a put the greater the probability that such a movement occurs.

Comparing prices implied by the equilibrium model to those calculated with standard BR or BS models finds that the latter significantly overprice both at-the-money calls and puts. This is due to the fact that option prices rise in volatility (positive vega) and the BS model, based on a standard geometric Brownian motion process, does not "factor out" that part of volatility due to irregular, discrete jumps. While the jumps add to overall volatility, weighting their occurrence by their probability significantly reduces their ultimate impact on the Monte Carlo option values. Consistent with the equity options (Merton; Naik and Lee; Bakshi, Cao and Chen) and foreign-exchange options literatures (Bates 1991; Jorion), the results in table 4 show that factoring out small probabilities of extreme fundamental events produces lower derivative price estimates. This is an important result given the nature of weather risks facing agricultural producers. 
Particularly in California's Central Valley, the band of normal fluctuation for both rain and temperature is quite small, but infrequent frosts or storms are the largest cause of economic damage. Some of this difference may also be due to the fact that the BS model does not account for mean reversion in the temperature index. In fact, Cao and Wei, Alaton, and Pirrong and Jermakayan all find that mean reversion is perhaps the most important feature of the weather index process. Dixit and Pindyck demonstrate the knifeedge nature of this result in the context of a real option model - while derivatives that admit the possibility of an explosive path away from the strike price will have a higher intrinsic value, low levels of mean reversion (as our data show) mean that volatility plays a greater role in determining the expected payoff.

Neither the BR nor the BS models allow for time-varying volatility. Given that derivative prices rise in volatility, this result would suggest that ignoring the timedependent nature of volatility will produce lower, not higher, option prices. However, Hull and White show that a standard BS pricing model underprices options that are out of the money, whereas the derivatives in table 4 are priced at-the-money for comparison purposes. Hull and White's result is not unique to financial options as Myers and Hanson show that pricing models for options on agricultural futures that incorporate time-varying volatility provide better estimates (as measured by root mean square error) than do constant-volatility alternatives.

[table 4 in here]

The results in table 5 suggest that there is indeed a significant relationship between temperature and nectarine yields. For a weather derivative based on this CDD index to be a valuable risk management tool for Fresno County nectarine growers, the 
CDD index must be correlated with nectarine yields. However, the non-linear relationship suggests that non-typical trading strategies are appropriate. Namely, a grower should implement a straddle strategy by simultaneously buying a put and a call with strike CDD values set at the optimal level indicated by the yield model. A straddle would protect the grower from yield losses that result from excessively hot or cold temperatures over the critical growing period. Moreover, because this strategy involves the purchase of two options, the importance of minimizing the bid-ask spread through accurate pricing methods is apparent.

[table 5 in here ]

\section{Conclusions}

If weather derivatives are to achieve sufficient liquidity to be widely used revenue riskmanagement tools, then finding better pricing models is a necessary step. Defining "better" as a more accurate representation of the true value of the claim on a CDD index, an improved pricing model must consider both the complexity of the underlying weather process and the fact that weather is a non-traded asset. In creating such a model, this study adopts an equilibrium pricing approach based on the valuation framework developed by Lucas. The equilibrium pricing model is applied to weather data from Fresno, CA and used to calculate prices for both puts and calls on a CDD index. In doing so, the study also estimates the market price of risk over a range of risk aversion assumptions for both types of derivative.

Previous research into similar types of processes (electricity, stock prices, exchange rates) have found the usual geometric Brownian motion assumption to be 
inadequate. To investigate whether this is also the case for a daily termperature series, this study considers four alternative specifications and tests among them using a series of likelihood ratio tests. These tests show that the preferred model for average daily temperature in Fresno, CA, once it is corrected for seasonality and long-term warming trends, is a mean-reverting Brownian motion process with first-order autoregressive errors and a log-normally distributed jump term. This process is considerably more complex than the geometric Brownian motion that is typically used to model other processes on which financial derivatives are based.

The equilibrium simulation finds that misspecifying the underlying weather process can result in significant overpricing of derivatives based on a cumulative CDD index. Specifically, allowing for mean reversion, time-varying volatility and the fact that weather processes consist of discrete jump-diffusion rather than continuous diffusion processes each lead to lower weather derivative prices. More importantly, perhaps, estimating the market price of risk over a range of risk aversion parameters shows that the risk premium can be a significant part of the derivative price. Consequently, when riskneutral valuation cannot be applied, assuming the market price of risk is zero could result in significant pricing errors. Estimates of a simple hedging-effectiveness model also show that these derivatives have potential as viable risk management tools. 


\section{Footnotes}

${ }^{1}$ For example, a "cooling degree day (CDD)" is defined as the amount by which the average temperature on a given day exceeds $650 \mathrm{~F}$, or $\mathrm{CDD}=\max (\mathrm{T}-65,0)$, where $\mathrm{T}$ is the average temperature on a particular day. A "heating degree day (HDD)," on the other hand, is the amount by which the average daily temperature falls below $650 \mathrm{~F}$. Weather derivatives are typically based on accumulated CDDs or HDDs over a defined period.

${ }^{2}$ According to a 1998 survey of California growers, $46.9 \%$ of growers ranked weatherrelated risks as the most important they face, followed by $32.0 \%$ citing output price risk (Blank)

${ }^{3}$ Basis risk also arises from the fact that yields depend on both precipitation and temperature. Rain and heat are not perfectly correlated, nor are they linearly related to yields and market prices. Moreover, temperature varies continuously from region to region, whereas precipitation risk tends to be more localized. If weather risk derives from both sources, then collecting useable data and defining a relevant index may be important, yet potentially difficult.

${ }^{4}$ The temperature process is modeled directly here, rather than the CDD index, because calculating the CDD index introduces a truncation point that adds unnecessarily to the complexity of the underlying process. Moreover, any CDD, HDD or variation thereof, can be calculated from a simulated temperature process (Cao and Wei 2003). 
${ }^{5} \mathrm{~A}$ burn rate model assumes that a derivative price is equal to the present value of its expected payoff at expiry, where the expectation is calculated on the basis of historical data. In this sense, burn rate models are also termed "actuarial" pricing models.

${ }^{6}$ Although there are CDD and HDD futures contracts on the CME, these apply to only a handful of major metropolitan areas, so cannot be used to hedge weather risks for growers in Central California, or many other agricultural areas for that matter.

${ }^{7}$ This latter assumption is justified on the grounds that it is unlikely that the market portfolio can have any impact on the number of degree days, but it is not necessarily true that weather events do not impact the market portfolio.

${ }^{8} \mathrm{~A}$ GARCH process is more general and parsimonious than the $\mathrm{ARCH}$ used here (Hull and White). Although estimating a $\operatorname{GARCH}(1,1)$ model, for example, would be preferable, it would not converge in this problem. This is perhaps understandable given the demands that GARCH models make on the data, the complexity elsewhere in our model, and the fact that a GARCH process would likely explain many of the same innovations defined within the model as jumps.

${ }^{9}$ This assumption is more general than Ball and Torous (1985), who assume a jump size of mean zero. 
${ }^{10}$ This assertion can be proven. Assume the aggregate dividend is measured by a broad index of stock prices, and follows a stochastic process with growth rate $\mu_{\mathrm{m}}$ and volatility $\sigma_{\mathrm{m}}$, there is a risky asset with growth rate $\mu_{1}$ and volatility $\sigma_{1}$, and the risk-free return is $\mathrm{r}$. Hull shows that the market price of risk for any traded asset must be equal to its excess return above the risk-free rate normalized by its volatility, or $\lambda=\left(\mu_{1}-r\right) / \sigma_{1}$. However, by the capital asset pricing model (CAPM), this excess return is determined in the market as compensation for the risk the asset contributes to an otherwise well-diversified portfolio, which depends upon the correlation of returns to the asset and market return: $\mu_{1}-r=\left(\rho \sigma_{1} / \sigma_{m}\right)\left(\mu_{m}-r\right)$. If $\rho=0$, therefore, $\mu_{1}-r=0$ and $\lambda=0$ as well.

${ }^{11}$ As in the power example, this study only considers weather derivatives role as volume risk management tools, although prices may also be, albeit more loosely, associated with weather. Investigating this dual role may be a fruitful topic of future research in this area. 


\section{References}

Alaton, P., B. Djehiche, and D. Stillberger. "On Modelling and Pricing Weather

Derivatives.” Working paper, Department of Mathematics. KTH, Stockholm, SE. 2001.

Bakshi, G., C. Cao, and Z. Chen. "Empirical Performance of Alternative Option Pricing Models." Journal of Finance 52(1997): 2003-2048.

Ball, C. A., and W. N. Torous. "On Jumps in Common Stock Prices and Their Impact on Call Option Pricing." Journal of Finance 40(1985): 155-173.

Bates, D. S. "The Crash of '87: Was It Expected? The Evidence from Options Markets." Journal of Finance 46(1991): 1009-1044.

Bates, D. S. "Jumps and Stochastic Volatility: Exchange Rate Processes Implicit in Deutsche Mark Options.” Review of Financial Studies 9(1996): 69-107.

Black, F., and M. Scholes. "The Pricing of Options and Corporate Liabilities." Journal of Political Economy 81(1973): 637-659.

Black, F., and M. Scholes. "The Valuation of Option Contracts and a Test of Market Efficiency.” Journal of Finance 27(1972): 399-417. 
Black, F. “The Pricing of Commodity Contracts." Journal of Financial Economics 3(1976): 167-179.

Blank, S. C. "Managing Risks in California Agriculture.” Update: Agricultural and Resource Economics Summer(1998): 1-9.

Bollerslev, T. “Generalized Autoregressive Conditional Heteroscedasticity.” Journal of Econometrics 31(1986): 307-327.

Boyle, P. “Options: A Monte Carlo Approach.” Journal of Financial Economics 4(1977): $323-338$.

Cao, M., and J. Wei. "Pricing Weather Derivatives: An Equilibrium Approach.” Working paper, The Rotman Graduate School of Management, The University of Toronto, Toronto, Ont. October 1999.

Cox, J., and S. A. Ross. "The Valuation of Options for Alternative Stochastic Processes." Journal of Finance and Economics 3(1976): 145-166.

Cox, J., J. E. Ingersoll, and S. A. Ross. “An Intertemporal General Equilibrium Model of Asset Prices.” Econometrica 53(1985): 363-384. 
Davis, M. "Pricing Weather Derivatives by Marginal Value." Quantitative Finance 1(2001): 305-308.

Dischel, R.. “The Fledgling Weather Market Takes Off,” Applied Derivatives Trading Focus. (http://www.adtrading.com). 1998.

Dischel, B. “Black-Scholes Won’t Do.” Weather Risk Special Report. Energy and Power Risk Management. Risk Publications. October, 1998.

Engle, R. “Autoregressive Conditional Heteroscedasticity with Estimates of the Variance of United Kingdom Inflation.” Econometrica 50(1982): 987 - 1007.

Hilliard, J. E., and J. A. Reis. "Jump Processes in Commodity Futures Prices and Options Pricing." American Journal of Agricultural Economics. 81(1999): 273 - 286.

Hull, J. C., Options, Futures, and Other Derivatives, $5^{\text {th }}$ ed. Upper Saddle River, NJ: Prentice-Hall. 2002.

Hull, J. C. and A. White. "The Pricing of Options on Assets with Stochastic Volatilities." Journal of Finance 42(1987): 281 - 300.

Jarque, C. M., and A. K. Bera. "Efficient Tests for Normality, Homoscedasticity and Serial Independence in Regression Residuals.” Economics Letters 6(1980): 255-259. 
Jarrow, R. A., and E. R. Rosenfeld. "Jump Risks and the Intertemporal Capital Asset Pricing Model." Journal of Business 57(1984): 337-351.

Johnson, B., and G. Barz. "Selecting Stochastic Processes for Modelling Electricity Prices." Energy Modelling and the Management of Uncertainty London U.K.: Risk Books. 1999.

Jorion, P. “On Jump Processes in the Foreign Exchange and Stock Markets.” Review of Financial Studies 1(Winter 1989): 427-445.

Lucas, R. E. “Asset Prices in an Exchange Economy.” Econometrica 46(1978): 14291445.

Merton, R. C. “Option Pricing when Underlying Stock Returns are Discontinuous.” Journal of Financial Economics 3(1976): 125-144.

Myers, R. J., and S. D. Hanson. "Pricing Commodity Options When the Underlying Futures Price Exhibits Time-Varying Volatility." American Journal of Agricultural Economics 75(1993): 121-130.

Naik, V., and M. Lee. "General Equilibrium Pricing of Options on the Market Portfolio with Discontinuous Returns.” Review of Financial Studies 39(Winter 1990): 493-521. 
Nelken, I. "Weather Derivatives - Pricing and Hedging.” Super Computer Consulting, Inc. Mundelein, Illinois.

Pirrong, C., and Jermakyan, M. "Valuing Power and Weather Derivatives on a Mesh Using Finite Difference Methods.” Working Paper, Olin School of Business, Washington University. St. Louis, Missouri. June 1999.

Schwarz, G. "Estimating the Dimension of a Model." The Annals of Statistics 9(1978): 461-464.

Turvey, C. “A Pricing Model for Degree-Day Weather Options.” Working paper. Department of Agricultural Economics and Business. University of Guelph, Guelph, Ontario. July 2001.

Weather Risk Management Association. “The Weather Risk Management Industry: Survey Findings for April 2001 to March 2002.” with PricewaterhouseCoopers (http://64.125.144.31/librarydocs/bc51 wrma/public/file444.ppt). May 2003.

University of California - Davis. Cooperative Extension. "Sample Costs to Establish a Peach / Nectarine Orchard and Produce Peaches / Nectarines.” Davis, CA. 2000. 
West, J. "Benchmark Pricing of Weather Derivatives.” Working Paper. School of Finance and Economics. University of Technology, Sydney, Australia. December 2002.

Yoo, S. "Weather Derivatives and Seasonal Forecast." Working paper. Department of Applied Economics and Business, Cornell University, Ithaca, NY. January 2003.

Zeng, L. "Pricing Weather Derivatives.” Journal of Risk Finance Spring(2000): 72-78. 
Table 1. Summary of Fresno Weather Station Data: 1970 - 2001

\begin{tabular}{lcc}
\hline Statistic & $\boldsymbol{w}_{\mathbf{t}}{ }^{\mathrm{a}}$ & $\boldsymbol{w s}_{\mathbf{t}}$ \\
\hline Sample Size & 11,050 & 11,050 \\
Mean & 63.702 & -0.005 \\
Variance & 13.942 & 5.960 \\
Minimum & 27.000 & -16.301 \\
Maximum & 96.000 & 14.403 \\
Skewness (b $)^{\mathrm{b}}$ & $0.335^{*}$ & $-0.338^{*}$ \\
Kurtosis $\left(\mathrm{b}_{2}\right)$ & $-0.700^{*}$ & 0.277 \\
Chi-Square (CS) & $76.293^{*}$ & 35.009 \\
Jarque-Bera (JB) & $14.090^{*}$ & $7.877^{*}$ \\
Augmented Dickey-Fuller (ADF) & $75.631^{*}$ & $46.895^{*}$ \\
Durbin-Watson (DW) & $0.072^{*}$ & 2.072 \\
GARCH (1,1) & 0.174 & -.200
\end{tabular}

${ }^{a}$ In this table, $w_{\mathrm{t}}$ refers to the temperature series, in degrees Fahrenheit, $w s_{\mathrm{t}}$ to the temperature series adjusted for seasonality, linear time trend and third-order autoregression, $b_{1}$ is the coefficient of skewness, $b_{2}$ is the coefficient of excess kurtosis. Both $b_{1}$ and $b_{2}$ are asymptotically normally distributed, with zero mean under the null hypothesis. The standard deviation of $b_{1}$ is 0.128 and for $b_{2}$ is 0.255 . CS is a chi-square goodness of fit test for residual normality based on comparing the expected and observed distribution of residuals. Dividing the observations into 30 groups implies 27 degrees of freedom and a critical value of 40.11 at a $5 \%$ level of significance. JB is a Jarque-Bera Lagrange multiplier test statistic for residual normality and is calculated as: JB $=\mathrm{N}\left(\mathrm{b}_{1}{ }^{2} / 6+\mathrm{b}_{2}{ }^{2} / 24\right)$. The JB test statistic is chi-square distributed with two degrees of freedom, so the critical value is 5.99 at a $5 \%$ level. The ADF (unit root) test is an F-test for the joint significance of $\mathrm{H}_{0}: \alpha_{0}=\alpha_{1}=\alpha_{2}$ in the regression:

$$
\Delta w_{t}=\alpha_{0}+\alpha_{1} w_{t-1}+\alpha_{2} t+\sum_{i=1}^{p} \beta_{i} \Delta w_{t-i}+\mu_{t}
$$

with $p=3$ and $\mu_{\mathrm{t}}$ iid normal error terms. The critical value at a $10 \%$ level is $\mathrm{F}=4.03$. DW is the Durbin-Watson test statistic for first-order autocorrelation and has a critical lower bound of 1.758 for all processes at a $5 \%$ level of significance. The test for $\operatorname{GARCH}(1,1)$ errors is a t-test of the $\phi_{1}$ coefficient in the following conditional variance function:

$$
h_{t}=\gamma_{0}+\sum_{j=1}^{q} \gamma_{j} \epsilon_{t-j}^{2}+\sum_{j=1}^{p} \phi_{j} h_{t-j}
$$

where $p=1$ and $q=1$ reflects the $\operatorname{GARCH}(1,1)$ assumption.

\footnotetext{
${ }^{\mathrm{b}}$ A single asterisk indicates rejection of the null hypothesis at a $5 \%$ level
} 
Table 2. Weather Process Comparison: Fresno Air Terminal, ${ }^{\circ}$ F, 1970 - 2000

\begin{tabular}{|c|c|c|c|c|c|c|c|c|c|}
\hline \multirow[b]{2}{*}{ Parameter } & \multirow[b]{2}{*}{ Description } & \multicolumn{2}{|c|}{$\mathbf{B} \mathbf{M}^{\mathbf{a}}$} & \multicolumn{2}{|c|}{ MRBM } & \multicolumn{2}{|c|}{ MRBM-J } & \multicolumn{2}{|c|}{ MRBM-J-ARCH } \\
\hline & & Estimate $^{\mathrm{b}}$ & t-ratio & Estimate & t-ratio & Estimate & t-ratio & Estimate & t-ratio \\
\hline$\alpha$ & Drift term & -0.002 & -0.041 & 0.001 & 0.026 & $0.429 *$ & 6.729 & $0.461 *$ & 6.495 \\
\hline$\kappa$ & Rate of mean reversion & & & $0.501 *$ & 61.122 & $0.496 *$ & 62.099 & $0.490 *$ & 54.526 \\
\hline$\lambda$ & Poisson arrival rate & & & & & $0.722 *$ & 74.815 & $0.819 *$ & 7.913 \\
\hline$\phi$ & Magnitude of jump (\%) & & & & & $-0.704 *$ & -6.908 & $-0.605^{*}$ & -7.047 \\
\hline$\sigma^{2}$ & Variance of continuous part & $21.370 *$ & 70.578 & $15.996 *$ & 74.270 & $9.130 *$ & 29.947 & & \\
\hline$a_{1}$ & $\mathrm{ARCH}$ intercept & & & & & & & $6.543 *$ & 11.780 \\
\hline$a_{2}$ & ARCH slope & & & & & & & $0.079 *$ & 8.720 \\
\hline$\delta^{2}$ & Variance of jump & & & & & $10.782 *$ & 17.779 & $9.143 *$ & 11.991 \\
\hline LLF & & $-32,502.4$ & & $-30,906.7$ & & $-30,263.6$ & & $-30,118.3$ & \\
\hline $\mathrm{LR}^{\mathrm{c}}$ & & $4,768.200 * *$ & & $788.400 * *$ & & $145.300 * *$ & & & \\
\hline $\mathrm{CS}$ & & 27.121 & & 20.739 & & 19.496 & & 21.624 & \\
\hline $\mathrm{JB}$ & & 3.494 & & 4.054 & & 3.787 & & 3.713 & \\
\hline $\mathrm{b}_{1}$ & & 0.039 & & -0.193 & & -0.180 & & -0.178 & \\
\hline $\mathrm{b}_{2}$ & & 0.496 & & 0.366 & & 0.369 & & 0.365 & \\
\hline DW & & 3.071 & & 2.312 & & 2.321 & & 2.330 & \\
\hline
\end{tabular}

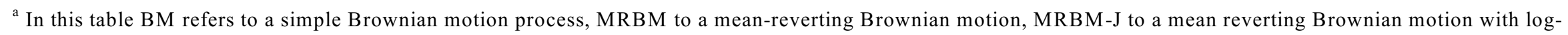
normal jumps, and MRBM-J-ARCH to a MRBM-J process with auto-regressive, conditionally heteroscedastic errors.

${ }^{\mathrm{b}}$ A single asterisk indicates rejection of the null hypothesis that the parameter equals zero at a $5 \%$ level of significance.

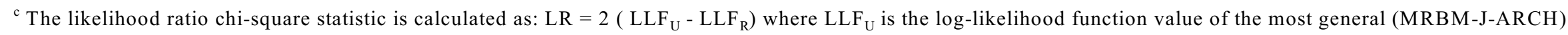

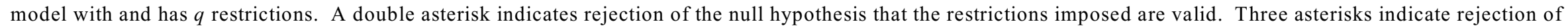

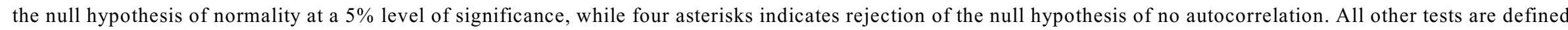
in a footnote to table 1. 
Table 3. Aggregate Dividend Process Estimates: Fresno County Personal Consumption Expenditure: 1970 - 2000 and Temperature Process Residuals

\begin{tabular}{|c|c|c|}
\hline Variable $^{\mathrm{a}}$ & Estimate & t-ratio \\
\hline Constant & $4.997 *$ & 12.330 \\
\hline$\epsilon_{\mathrm{t}}$ & $4.931 *$ & 1.779 \\
\hline$\epsilon_{\mathrm{t}-1}$ & $6.792 *$ & 2.420 \\
\hline$\epsilon_{\mathrm{t}-2}$ & $5.287^{*}$ & 1.921 \\
\hline D.W. & 1.719 & \\
\hline $\mathbf{R}^{2}$ & 0.293 & \\
\hline RESET - 2 & 0.076 & \\
\hline RESET - 3 & 0.994 & \\
\hline RESET - 4 & 0.700 & \\
\hline
\end{tabular}

${ }^{\mathrm{a}}$ Dependent variable is $\Delta y_{\mathrm{t}}=$ change in annual personal consumption expenditure for Fresno County, CA. Independent variables are annual average residuals from estimates of preferred weather process in table 2: $\epsilon_{t}=w_{t}-\hat{w}_{t}$, where $\hat{w}_{t}$ is the corrected (for seasonality, autoregression and drift) temperature value for day $t$. Critical values for the RESET tests are: $\mathrm{F}_{5 \%, 1}$, ${ }_{23}=4.28, \mathrm{~F}_{5 \%, 1,22}=4.30$, and $\mathrm{F}_{5 \%, 1,21}=4.32$ for the RESET - 1, RESET - 2, and RESET - 3 tests, respectively. A single asterisk indicates significance at a $10 \%$ level. Constant term is scaled by a factor of $10^{6}$ to facilitate presentation. 
Table 4. Weather Derivative Monte Carlo Equilibrium Price Estimates: Fresno County

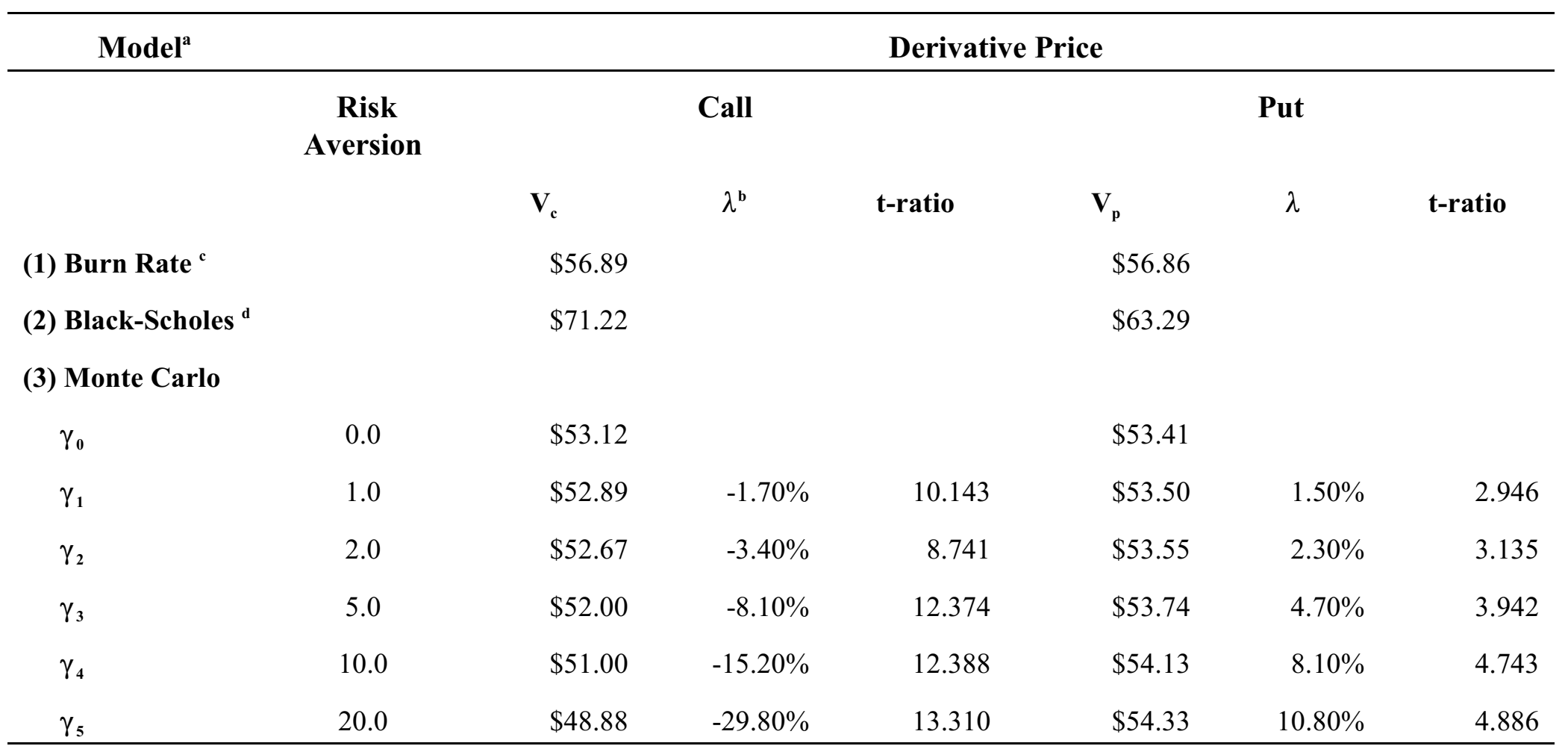

${ }^{\text {a }}$ In this table, all derivative price simulations use the preferred (MRBM-J-ARCH) weather process parameter estimates in table 3, a risk-free rate of $3 \%$, a common strike CDD equal to the long-run average of 1047.4, a tick-rate of $\$ 1.00$ per accumulated CDD and a time-period of 92 days (May $1-$ July 31 ). All simulations use 10,000 replications of the normal and Poisson deviates. All rates of return are expressed on a annual basis.

${ }^{b}$ The null hypothesis is that the market price of risk is zero. For each derivative type (call or put), the first row $\left(\gamma_{c}\right)$ under the "Monte Carlo" heading is the price with a zero market price of risk, while subsequent rows assume a non-zero market price of risk consistent with the associated coefficient of relative risk aversion. The next column gives the implied market price of risk calculated by comparing each derivative price with the risk-neutral benchmark.

c "Burn rate" prices are calculated such that each derivative is priced to be actuarially sound on a historical basis. That is, option prices are the present value of expected payoffs at expiry given historical probabilities and the expected payoff relationships for a call: $C=\max (\mathrm{CDD}-K, 0)$ and a put: $P=\max (K-\mathrm{CDD}, 0)$, where $K$ is the strike value.

${ }^{\mathrm{d}}$ Black-Scholes prices are calculated using the Black-Scholes options pricing formula with assumed historical volatility and average accumulated CDD value at expiry. 
Table 5. Estimates of Hedging Effectiveness: Weather Impact on Yield

\begin{tabular}{lcc}
\hline \multicolumn{3}{c}{ Nectarine } \\
\hline \multicolumn{1}{c}{ Variable $^{\mathrm{a}}$} & Estimate & t-ratio \\
\hline Const. & $-5.025^{*}$ & -2.009 \\
$\boldsymbol{t}$ & -0.044 & -1.549 \\
$\boldsymbol{w}_{\mathbf{1}}$ & $27.471^{*}$ & 2.956 \\
$\boldsymbol{w}_{\mathbf{1}}{ }^{2}$ & $-11.028^{*}$ & -2.628 \\
\hline RESET - 1 & 3.734 & \\
RESET - 2 & 2.438 & \\
RESET - 3 & 1.547 \\
\hline $\mathbf{R}^{2}$ & 0.618 \\
\hline
\end{tabular}

${ }^{\mathrm{a}}$ In the above table, $t$ is a linear time-trend variable and $w_{1}$ is the value of the CDD index at the end of the sample period. Yield is defined as tons per acre. Critical values for the RESET test at a 5\% level are: $\mathrm{F}_{1,14}=4.60, \mathrm{~F}_{1,13}=4.67, \mathrm{~F}_{1,12}=$ 4.75 . 